\title{
High performance protocol architectures - HIPPARCH project and workshop
}

\author{
Jon Crowcroft \\ Department of Computer Science, UCL, Gower St. London, WC1E 6BT, UK \\ E-mail: j.crowcroft@cs.ucl.ac.uk
}

\begin{abstract}
The objective of the HIPPARCH project is to study and design high performance communication architectures and implementations, based particularly on the "Application Level Framing" and "Integrated Layer Processing" concepts. The HIPPARCH project holds annual workshops at which papers from leading communications research centers are presented. The papers in this issue of JHSN represent early results of the project and other groups engaged in similar research.
\end{abstract}

\section{Introduction}

HIPPARCH is an EC ESPRIT Basic Resarch project which began in january 1994 and is tasked with establishing a novel architectural design for communications protocols to be used for distributed applications over high speed networks. The project partners, INRIA, SICS, UCL and UTS, are all longstanding members of the research community working on improving our understanding and implementation of communications systems.

The requirement for a new architecture is clear. Traditional layered protocol architectures such as the ISO OSI model (see for example [3]) and the ARPA Internet model (see for example [4]), are reaching the very end of their extended lifetimes.

The problem can partly be blamed on an artificial separation of concerns in layers that represented interfaces between different service providers: The service/protocol concept derives historically from the model X.25 originally presented to the user of a network as interface. There would be a link, network, transport and session provider, perhaps all of which would be different potential vendors. This absurd extreme view of a potential "market" in layers in the stack has proved one thing: The costs of such a market in inefficiency mean buyers go to other markets - the workable market appears to be in three layers: end system hardware and operating systems; end systems communications stacks; finally transmission networks. HIPPARCH concentrates its efforts on the second of these, to focus on end-to-end transmission control mechanisms and architecture.

We are seeing a frantic flurry of effort piggybacked on the IPng effort to introduce a new architecture. Examples of such attempts include [5] and [6]. Some of these have led to partial attempts to address transport protocol problems as well [2]. While these address the network layer, they do not provide an integrated approach to designing the whole stack.

We have adopted "Application Level Framing" and "Integrated Layer Processing" as intuitively reasonable guiding principles for selecting a new architecture that does address whole protocol stack design.

In the initial year, we have carried out a number of tasks to deconstruct the problem. These fall roughly under the following headings:

Adaptable transmission control mechanisms. Networks and End systems are and will probably always be heterogeneous. We should design systems that can operate for a large range of applications as well as TCP does now for a small one. 
Novel implementation techniques. We want to gather as many manual implementation techniques together as the basis of our decision on an architecture - we want to maximise the "gene pool diversity" of the communications eco-system we are trying to build. In particular, we want to avoid pitfalls present in hidden assumptions about operating system structures (Unix, OS/2, Windows NT, etc) and about processor/memory architectures (uni versus multi, shared versus distributed memory) in the end system. In general, the final goal is a novel communication support architecture. This is not limited to implementation architecture, but to gain more from implementation techniques, the architecture itself has to be modified.

Experiences with ALF/ILP. While we seek new techniques, we would like to gain as much experience as possible using these particular guiding principles.

Tools and description languages for protocol implementation. Initially, a lot of our efforts have involved manual programming of our new stacks. However, the objective is to eventually provide descriptions of application requirements, and automatically synthesize new protocols and protocol stacks to support the applications. To this end, initially, we wish to describe protocols and modules, and automatically generate working systems. Later this will permit verification/[8] and validation [8].

\section{Papers in JHSN}

There are eight papers which fall into 4 natural groups of 2 :

On the practical advantages of using a formal language approach:

Automated design of communication protocols using ESTEREL. C. Diot, R. De Simone and C. Huitema. INRIA Sophia Antipolis (F).

OpParIm: a method and tool for optimized parallel protocol implementation. Stefan Leue and Phillippe Oechslin. University of Waterloo (CA), SFIT (CH).

On flexibility and configuration:

Design and implementation of flexible User Protocol Interface. B. Metzler and I. Miloucheva. TUB. Berlin (D).

The performance of configurable protocols. A. Richards, R. De Silva, A. Seneviratne, M. Fry and A. Fladenmuller. UTS. Sydney (AU), Université Pierre et Marie Curie (F).

On the system structure and its influence on performance:

Evaluating the impact of ALF on communication subsystems design and performance. I. Chrisment and C. Huitema. INRIA. Sophia Antipolis (F).

From the partial order connection concept to partial order multimedia transport connections. C. Chassot, M. Diaz and A. Lozes. LAAS. Toulouse (F).

On high performance implementation techniques:

Demultiplexing on the ATM adapter: experiments with Internet protocols in user space. E.W. Biersack and

E. Rütsche. EURECOM (F).

Increasing communication performance with a minimal-copy data path supporting ILP and ALF. B. Ahlgren, P. Gunningberg and K. Moldeklev. SICS (S), Uppsala University (S), Telenov Research (N).

\section{Workshop papers}

The workshop features sixteen papers from all around the communications community. We received papers addressing all the aspects of the research we wished and more. As well as Integrated Layer Processing and Application Layer Framing, work is described on intelligent buffering systems to minimise copies, the impact of parallel systems on protocol architectures, the impact of multicast and multi-party communications in general. 
Languages for protocol development and configuration, operating system support, and general system engineering c.nsiderations are all addressed.

The Workshop had other papers of value which we have neither time nor space to present here:

Application level framing.

Chairman: Per Gunningberg. SICS (S).

1-Integrated layer video decoding and application layer framed secure login-general lessons from two or three very different applications.

A. Ghosh, M. Handley, Z. Wang and J. Crowcroft. UCL. London (UK).

7-High performance event filtering for dynamic multipoint applications.

D.C. Schmidt. Washington University. St. Louis (USA).

Configuration of protocols.

Chairman: Aruna Seneviratne. UTS (AU).

8-Efficient configuration of protocol software for multiprocessors.

S. Fischer and W. Effelsberg. University of Mannheim (D).

9-PROCOM: A protocol configuration manager in the function-based communication subsystem.

B. Stiller. University of Cambridge (USA).

Special Guest: Second generation multimedia networking and endworking implications

David Tennenhouse. MIT (USA). Through MBone.

Efficient implementations

Chairwoman: Martina Zitterbart. University of Karlsruhe (D).

11-High performance protocol implementations in the Scout operating system - Invited paper.

S. O'Malley. University of Arizona (USA).

System integration

Chairman: Michel Diaz. LAAS (F).

14-Towards integrated QOS management.

C. Schmidt and M. Zitterbart. University of Karlsruhe (D).

15-Evaluating crucial performance issues of protocol configuration in $\mathrm{Da} \mathrm{CaPo}$.

T. Plagemann. University of Oslo (N). B. Plattner, ETH (CH).

16-System architecture considerations for efficient protocol implementations.

P. Boonchai-Apsit, M. Fry and A. Seneviratne. UTS (AU).

Closing Debate: The future of protocol architectures.

Moderator: Jon Crowcroft. 


\section{References}

[1] D.D. Clark and D.L. Tennenhouse, Architectural considerations for a new generation protocols, Computer Communication Review 20(4) SIGCOMM '90, (September 1990), 200-208.

[2] Van Jacobson, Multimedia conferencing on the Internet, Tutorial Notes, http://www.cs.ucl.ac.uk/mice/van/sigcomm94.html, SIGCOMM '94, September 1994.

[3] IEEE Special Issue on Open Systems Interconnection (OSI), Proc. IEEE 71(12) (December 1983), 1329-1488.

[4] B.M. Leiner, R.H. Cole, J.B. Postel and D. Mills, The DARPA Internet Protocol Suite Proceedings INFOCOM '85, IEEE, March 1985.

[5] R. Braden, D. Clark and S. Shenker, Integrated services in the Internet architecture: an overview SRI Intl, RFC 1633, September 1994.

[6] J. Noel Chiappa, IPng Technical Requirements of the Nimrod Routing and Addressing Architecture Work in progress, draft-chiappaipng-nimrod-arch-00.txt, Jul. 1994.

[7] G.J. Holzmann, Design and Validation of Computer Protocols, Prentice-Hall, 1991.

[8] B. Meyer, Object-Oriented Software Construction, Prentice-Hall, 1988. 